\title{
Delivering DNA origami to cells
}

\author{
Dhanasekaran Balakrishnan ${ }^{\ddagger 1,2}$, Gerrit D Wilkens ${ }^{\ddagger 1,2}$ \& Jonathan G Heddle *,1 \\ ${ }^{1}$ Bionanoscience \& Biochemistry Laboratory, Malopolska Centre of Biotechnology, Jagiellonian University, Gronostajowa 7A, \\ 30-387 Krakow, Poland \\ ${ }^{2}$ Postgraduate School of Molecular Medicine; Żwirki i Wigury 61, 02-091 Warsaw, Poland \\ *Author for correspondence: jonathan.heddle@uj.edu.pl \\ $\ddagger$ Authors contributed equally
}

DNA nanotechnology research has long-held promise as a means of developing functional molecules capable of delivery to cells. Recent advances in DNA origami have begun to realize this potential but is still at the earliest stage and a number of hurdles remain. This review focuses on progress in addressing these hurdles and considers some of the challenges still outstanding. These include stability of such structures necessary to reach target cells after administration; methods of cell targeting and uptake; strategies to avoid or escape endosomes and techniques for achieving specific subcellular localization. Finally, the functionality that can be expected once DNA origami structures reach their final intracellular targets will be considered.

Lay abstract: The DNA origami technique allows 'nanorobots' to be made from DNA. These have the potential to work as 'smart' drugs but only if they can be successfully delivered to cells. Here, we review progress in achieving this goal.

First draft submitted: 15 November 2018; Accepted for publication: 23 January 2019; Published online: 22 March 2019

Keywords: cell delivery $\bullet$ cell targeting $\bullet$ DNA origami $\bullet$ DNA nanotechnology $\bullet$ nanorobots $\bullet$ smart medicines

DNA nanotechnology can trace its roots back to Nadrian Seeman, who proposed that by introducing unique sequences in the arms of Holliday junctions, immobile branched DNA junctions could be constructed, which could be used as building blocks to assemble higher order structures such as polyhedra [1]. Following his theoretical observations, Seeman and colleagues first constructed and synthesized an immobile 4-arm junction [2], later followed by branched structures of up to 12 arms [3,4]. Already envisioned in Seeman's initial paper on immobile junctions [1], designed 3D nanostructures were first demonstrated in 1991 where a cube-like shape was assembled from DNA oligonucleotides [5]. Other polyhedral structures and also lattices were soon to follow [6,7] with a DNA tetrahedron first being demonstrated by Goodman et al. [8,9]. Because of their relatively facile single step assembly and high yield, DNA tetrahedrons have been an early and widely utilized DNA nanostructure for drug loading and cell targeting/delivery [10-13]. DNA origami itself was first demonstrated in 2006 as a method for designing and constructing 2D shapes [14]. The concept of DNA origami is amply explained in several other review papers [15-18]. In brief, it is typically constructed using a single-stranded (ss) template DNA of approximately $7 \mathrm{~Kb}$ in length. In most cases, this is sculpted into the designed shape using approximately 200 staple strands that are complementary to distal parts of the template strand and can bring them into close proximity ('stapling'). With the help of software [19] complex 2D and 3D structures can be designed and made with the construction process typically consisting simply of mixing the DNA strands together and then annealing them [20]. Many potential applications for DNA origami have been proposed from materials to drug delivery [21,22] and it has shown potential as a means to interface with cells [23,24]. A growing area of interest is cellular delivery of DNA nanostructures [25-29], which we discuss in this review, focusing primarily on DNA origami structures, and including additional examples from other non-origami DNA nanostructures wherever helpful.

Delivery of molecules and nanoparticles to cells involves overcoming many challenges (Figure 1) depending on both the cell type and the nature of the delivered particle. For animal cells (considered here) there are a number of possible uptake pathways. It is typically the case that for cells whose in vivo role includes uptake of foreign particles 


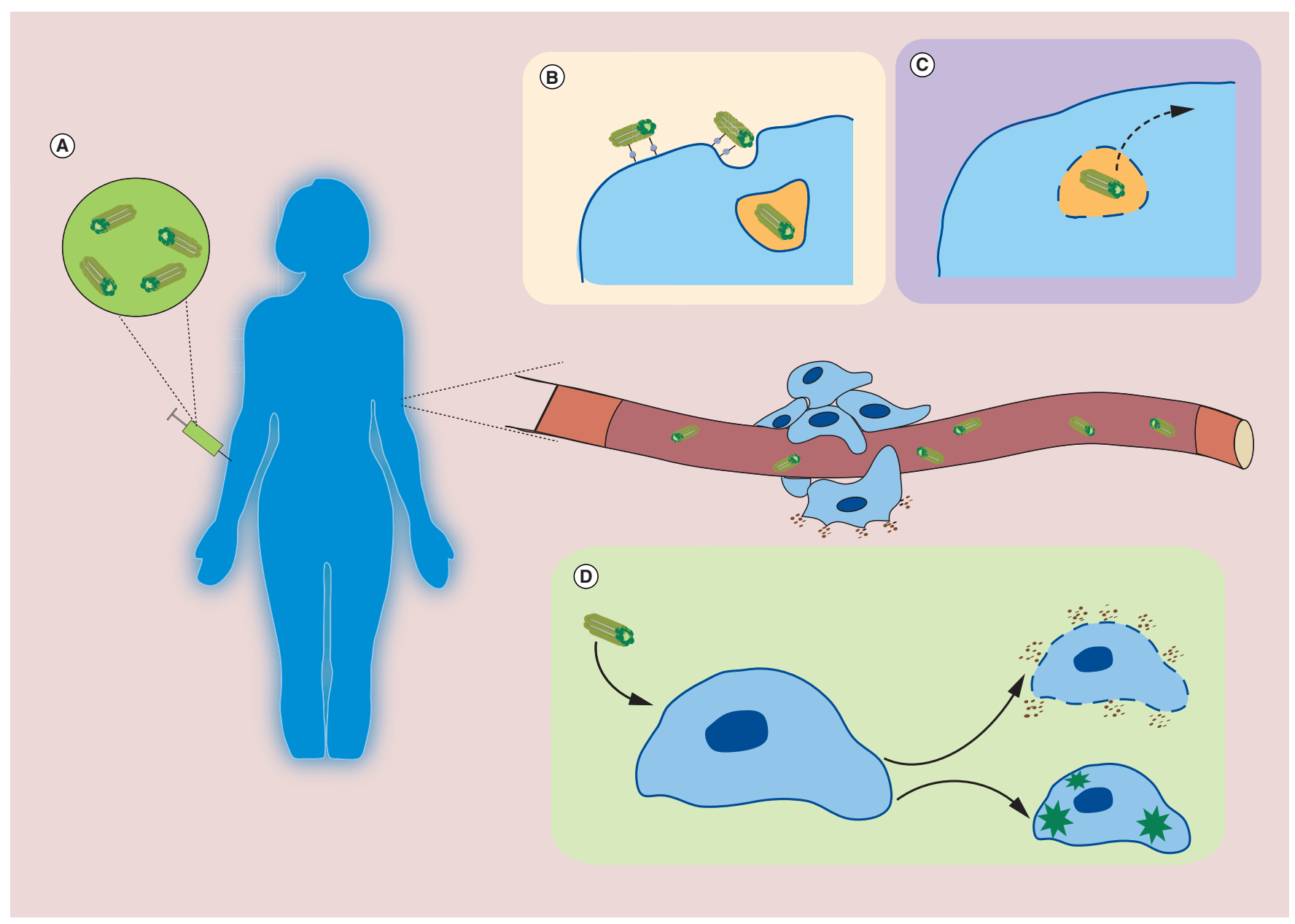

Figure 1. Challenges in the cellular delivery of DNA origami structures. (A) After being administered to the body, DNA origami nanodevices have to cope with the encountered in vivo conditions such as clearance for example, by liver, spleen or kidney or the mononuclear phagocyte system, (B) find or accumulate at and often internalize into the targeted cell types, (C) escape or release loaded cargo from the endosome and (D) finally execute the proposed function (e.g., cell lysis, in vivo imaging, etc.).

for example, processing and presenting to the immune system, such as macrophage, monocytes and dendritic cells [30], delivery may not require special targeting or cell penetration designs as long as they are not outside of the preferred size range (50-200 $\mathrm{nm}$ [31]). These cell types are involved in processing and presentation to the immune system and delivery to them can be useful if the purpose of the delivered particle is immunomodulatory, for example, to provide antigens for antigen display. In contrast, many other cell types are more selective in the particles that are taken up and therefore mechanisms to target them and stimulate entry/internalization need to be considered and included in the nanoparticle design. Precise targeting is of course an advantage for therapeutic delivery where it is desirable to affect only a specific cell type to avoid side effects. For smaller particles and molecules, passive diffusion through the cell membrane may be possible depending on their charge, as is the case for carbon nanotubes, which have been shown to diffuse through the cell membrane to the cytoplasm in a process not dependent on endocytosis [32].

Characteristically, once delivered into a cell, a particle will be in an intracellular compartment (typically an endosome) and will need to release a therapeutic molecule that can escape from it, or if the whole nanoparticle is required for functionality, will have to escape in its entirety from the compartment. Once in the cytoplasm, the particle must be able to localize to the required subcellular location and overcome any additional barriers (e.g., the mitochondrial membrane, nuclear membrane, etc.). Finally, once at the target site it must carry out its required function, which in the case of DNA origami could include relatively complex, programmable 'robotic' mechanisms. Each of these challenges are considered in turn in this review. 


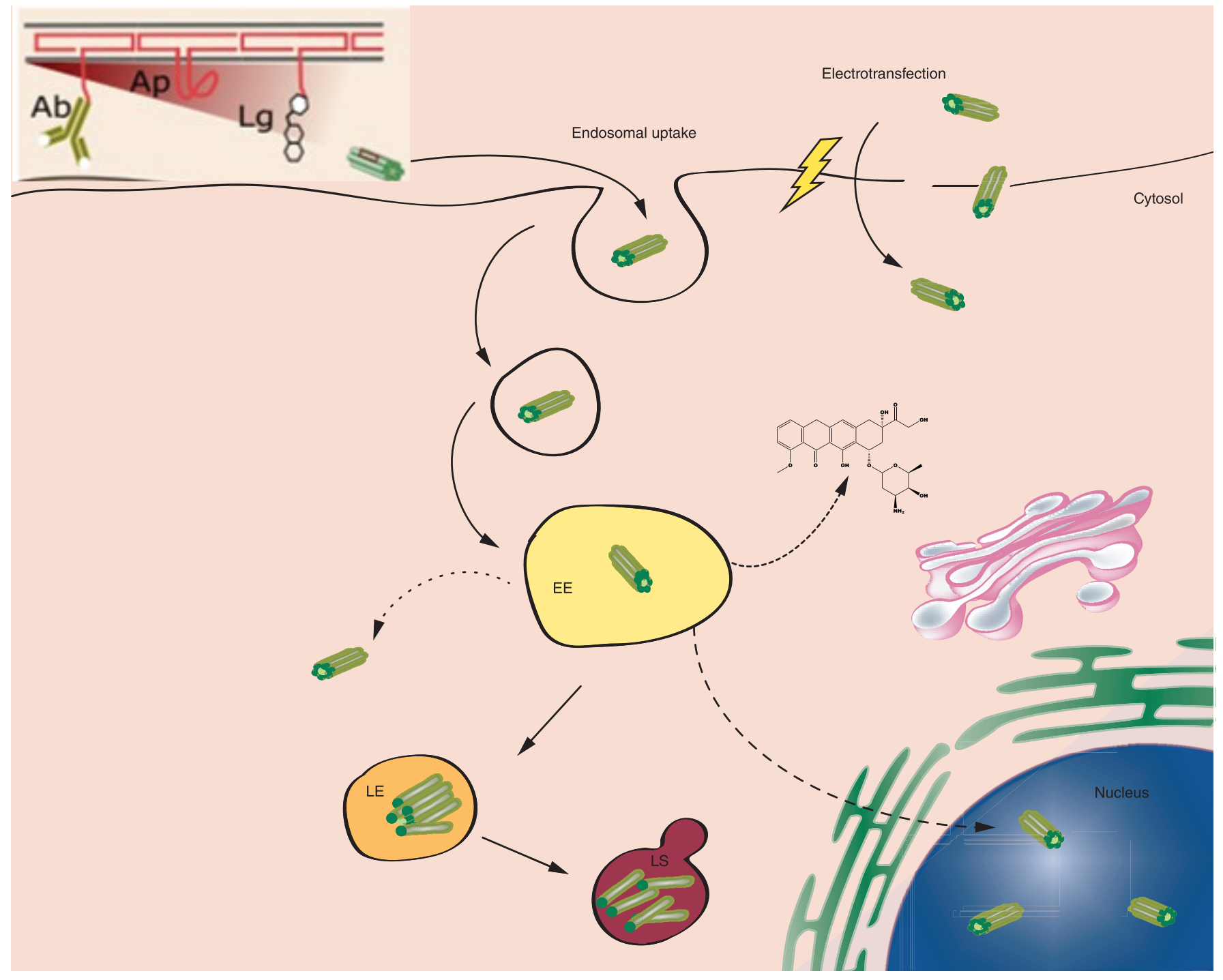

Figure 2. Cellular targeting and entry of DNA origami. DNA origami allows incorporation of functional groups such as ligands (e.g., folate), aptamers or antibodies to target specific cell types [42,43]. After uptake, DNA origami will, in most cases, be trapped in the endosomal compartment from where it has to escape or release a loaded cargo. To directly deliver DNA origami into the cytoplasm, electroporation can be used [40], where a strong electrical field ruptures the outer membrane and allows entry of the origami structures. Inset (top left) shows a detail of part of a DNA origami surface illustrating potential modifications.

Ab: Antibody; Ap: Aptamer; EE: Early endosome; LE: Late endosome; Lg: Ligand (e.g., folate); LS: Lysosome.

\section{Cell targeting \& uptake}

Once administered, a potential DNA origami-based therapeutic must survive the journey to the cell, must recognize and bind to the cell and must penetrate it (Figure 1). The first requirement for cell targeting is that a DNA origami structure can maintain integrity for long enough to be distributed to the target cell. For a typical in vivo delivery situation (i.e. intravenous administration) this means survival in the blood. Experiments with a DNA origami structure decorated with aptamers able to bind a protein diagnostic for malaria have shown that the structure remains intact and functional in blood plasma [33] while others have shown viability in living animals [21,34]. Once inside a target cell, the origami must once again survive long enough to carry out its function, something which has previously been tested in experiments where 2D and 3D shapes were demonstrated to be stable for $12 \mathrm{~h}$ in different cell lysates (CP-A, End1/E6E7, MCF-10A, HeLa and MDA-MB-231) [35].

For delivery to cells in vitro but also in vivo (Figure 2), direct delivery to the cytoplasm can be achieved using 
electrotransfection [36,37]. This employs a strong electric field which can strip away the $\mathrm{Mg}^{2+}$ counterions that typically stabilize the origami structure, potentially leading to loss of structural integrity. However, once formed, the ability of DNA origami structures to maintain integrity at low $\mathrm{Mg}^{2+}$ concentrations depends to large extent on the specifics of the individual superstructure with some requiring little or no $\mathrm{Mg}^{2+}$ [38,39]. As a more general strategy to overcome the dependence on $\mathrm{Mg}^{2+}$, folding of 3D DNA origami structures in absence of $\mathrm{MgCl}_{2}$ and presence of low spermidine concentrations has been developed [40]. Spermidine is a natural DNA-condensating agent and can thus mimic the properties of $\mathrm{MgCl}_{2}$ in the folding buffer. The resulting structures were shown to be stable and could be electroporated into Jurkat cells, whereas $\mathrm{MgCl}_{2}$-stabilized origamis were not internalized. In an alternative approach, a new algorithm was developed for designing DNA origami shapes with an open mesh structure that required fewer cations and these origamis were shown to be more stable in buffers mimicking physiological conditions [41].

In common with all therapeutics, it is necessary to be able to target DNA origamis to the required cell types so as to decrease potential side effects. Interestingly, it has been demonstrated that some drug-loaded origami shapes can effectively target and/or kill cancer cells even when not modified with specific targeting moieties [21,44], something which in vivo, is most likely due to enhanced permeability and retention effects [21]. Indeed, simply altering the size and shape of the DNA origami can affect uptake fate [45].

The obvious way to achieve targeting is to include, within the DNA origami structure, a ligand that is able to specifically recognize and bind to an identifying surface molecule on the cell type of interest (Figure 2). One way to achieve this is to use DNA aptamers; DNA sequences that are analogous to antibodies and which can be produced in vitro using the SELEX technique [46,47]. A number of aptamers have been produced including against thrombin [48] and lactate dehydrogenase from Plasmodium falciparum [49] with both having been shown to be able to function as part of DNA origami structures [33,50-52] and general principles for successful aptamer integration are beginning to be understood [53]. In the field of cell delivery, aptamers have been used to target cancer cells. For example, targeting of the MUC1 receptor overexpressed on many cancer cell lines has been shown in work in which a DNA origami nanosphere was produced that consisted of two hemispheres. The sphere was modified with an aptamer based 'lock and key' that kept it in a closed conformation but could open it after binding to MUC1. The sphere was proven to be stable under various conditions, ( $\mathrm{pH}$ : $4-8$, temperatures up to $56^{\circ} \mathrm{C}$, incubation for $24 \mathrm{~h}$ in serum containing DMEM or $6 \mathrm{~h}$ in MCF-7 and MDA-MB-231 cell lysates) suggesting potential applicability to cells. Open and closed states of the sphere were assessed using FRET and after addition of cell lysate of cancer cell lines having high and low expression of MUC1 to the origami sphere, it was shown that cell lysate of strongly MUC1 expressing cell lines led to fast opening of the sphere, whereas the other cell type did not [54]. The concept of using aptamers extends beyond DNA origami to other DNA nanostructures for example, the sgc8 aptamer [55] that binds to PTK7 [56] has been tethered to a DNA 'nanotrain' consisting of two short self-polymerizing DNA building blocks. The structure was shown to specifically internalize into PTK7-expressing target CEM cells but not on non-target Ramos cells. Doxorubicin (dox) could be intercalated into the structure with the resulting complex showing cytotoxicity toward CEM cells (but not to Ramos cells), whereas free dox non-selectively affected viability of both CEM cells and non-target cells. In a mouse tumor model, the loaded structure resulted in reduced tumor growth/increased survival rate with better efficiency compared with dox alone and reduced side effects [42].

Other cell targeting approaches include the inclusion of folate for targeting the folate receptor which is overexpressed on a number of cancer cell types [57] and has been demonstrated on smaller DNA nanostructures (DNA tetrathedron) for delivery of SiRNA to HeLA cells [11].

Understanding uptake pathways of DNA origami in the cell is important and here again DNA tetrahedra have shown the way: in the work of Liang et al. [12] they were labeled with Cy5 and added to HeLa cells. The fate of the DNA was followed by single particle tracking which showed that they entered the cell but not the nucleus. Colocalization studies in which the lipid membrane was also stained suggests DNA fused with the cell membrane and were taken up by endocytosis. Lack of internalization at $4{ }^{\circ} \mathrm{C}$ suggested an energy dependent uptake. Cytochalasin, which inhibits non-receptor-mediated uptake had no effect, suggesting uptake was receptor mediated. In contrast, methyl-beta-cyclodextrin which inhibits caveolin-dependent uptake, caused a large reduction in uptake of the DNA. Sucrose, which inhibits clathrin-dependent uptake had no effect, suggesting the DNA is taken up by a caveolin-dependent process. Tracking showed some tetrahedra remained static, others moved large distances. Treating cells with nocodazole (a tubulin depolymerizer) stopped large distance movement, while cytochlasin B (an actin depolymerizer) had no effect, suggesting a transport via kinesin and dynein. The ultimate destination 
was shown to be lysosomes (by co-staining with lysotracker). In order to show that lysosomal escape was possible, the tetrahedrons were modified with a peptide having a nuclear localization signal (labeled with TAMRA, while tetrahedron was labeled with Alexa 488). After incubation, cells were stained with Hoechst 33342 to stain the nucleus. Optical sectioning showed the tetrahedrons to be localized in the nucleus.

When no specific receptor or uptake pathway is targeted, then it may be necessary for the DNA origami particle to have a cell membrane penetrating ability to deliver a therapeutic cargo to the interior. Strategies to achieve this include modification of DNA origami with cell-penetrating peptides (CPPs) and encapsulation into larger structures, which may be able to more effectively penetrate cells. In the latter case, rectangular DNA origami has been coated with virus capsid proteins (CP) from cowpea chlorotic mottle virus, which results in the formation of $\mathrm{CP}$-origami complexes with different morphologies: the highly-flexible DNA rectangle could adopt either a rolledup or a completely encapsulated form depending on the relative amount of protein present [58]. The transfection rates of these CP-origami complexes into the human cell line HEK293 were measured using confocal microscopy and were shown to be significantly higher than for naked origamis and, importantly, no detectable toxicity was observed. In addition, cellular transfection rates progressively improved with increasing amounts of added CPs. The use of CPPs is another method to overcome the cell membrane. In one example [43], a tile based highly branched DNA nanostructure was created and decorated with cytosine-phosphate-guanine (CpG) loops and the cell penetrating peptide TAT [59]. CpG was attached in loop strands to the end of the branches, in order to increase resistance of the structure against nuclease restriction. Analysis by agarose gel electrophoresis showed sharp bands after $8 \mathrm{~h}$, and still visible bands after $24 \mathrm{~h}$ incubation in 10\% fetal bovine serum, whereas structures lacking the $\mathrm{CpG}$ loop degraded after $24 \mathrm{~h}$ of incubation. Cell uptake of the structure in RAW264.7 macrophage-like cells was studied using FACS and fluorescence microscopy. TAT protein was shown to strongly increase cellular entry and the structure was found to be localized in endosomal compartments. Immuno-stimulating effects were determined by measuring TNF- $\alpha$ and IL- 6 release, which showed that the structure decorated with both CpG and TAT was most effective in immunostimulation.

CPPs have also been used in conjunction with DNA origami, gold nanoparticles (AuNPs) and drug [60]: AuNPs were decorated with short single-stranded DNA (ssDNA), which were extended by rolling circle amplification (RCA) using a circular DNA as a template. The single-stranded extensions were then folded into belts that extended from the AuNPs. DLS showed an average hydrodynamic radius of around $84 \mathrm{~nm}$ for the AuNP with RCA extended ssDNA and of around $573 \mathrm{~nm}$ for the folded origami structures, indicating that higher ordered structures are formed where the origami belts extend in every direction. The origami was then functionalized by introducing biotin into the ssDNA (using biotin-dATP) and one of the staples, and simultaneously loaded with quantum dots and CPPs. The structure was successfully delivered into U87 MG cells (glioblastoma cell line) and showed strong fluorescence, whereas origami lacking the CPPs could not internalize. The AuNP-origami, the AuNP-RCA and AuNPs with the single-stranded primer used for RCA (AuNP-primer) were loaded with dox and functionalized with CPPs and used to induce cell death. The AuNP-origami showed the highest killing efficiency compared with AuNP-RCA, AuNP-primer, free dox plus CPPs, free dox plus CPPs and AuNPs, as well as the folded and dox/CPP loaded origami structure, RCA not bound to AuNPs and the fully assembled AuNP-origami structure without dox loaded. This may be because the dox-loading capacity is higher than for the other particles, but the effect may also be because the origami structure leads to slow degradation and thus slower drug release in the cell.

As well as structural integrity of the origami en route to the cell, there is also the possibility of undesirable immune stimulation. This can be overcome by coating the DNA origami structure with immune-silent materials (Figure 3). Lipid encapsulation has been reported for a DNA origami nano-octahedron [61] in which helper strands were base-paired to handle strands on the outer surface of the structure and these helper strands were modified with a terminal lipid. Enveloping the DNA nanostructure in lipid bilayers had a number of effects: nuclease protection, significantly decreased immune activation and significantly increased bioavailability. Encapsulation in the lipid almost completely inhibited uptake by immune cells while in vivo kinetics showed that the half-life was much increased compared with non-encapsulated origami and oligonucleotides. Both oligonucleotides and the no-nencapsulated origami were renally cleared indicating rapid breakdown (only sub- $6 \mathrm{~nm}$ particles are renally cleared). Interestingly this contrasts with other reports [21] showing naked origami structures (not encapsulated) to be stable in vivo. It is suggested that this may be dependent on the DNA origami structure as well as the possible effect of buffer components used in stability tests, on nuclease activity. Biodistribution of the lipid-encapsulated DNA origami was assessed, with liposomes alone and the encapsulated origami both showing similar results, being 
(A)

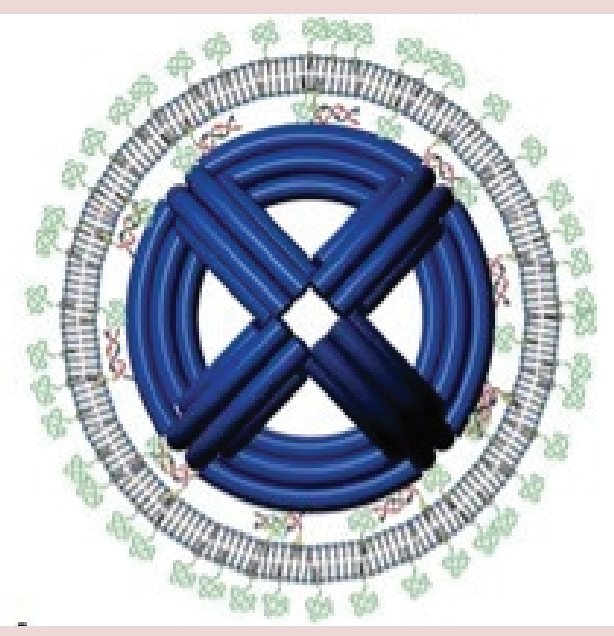

(B)

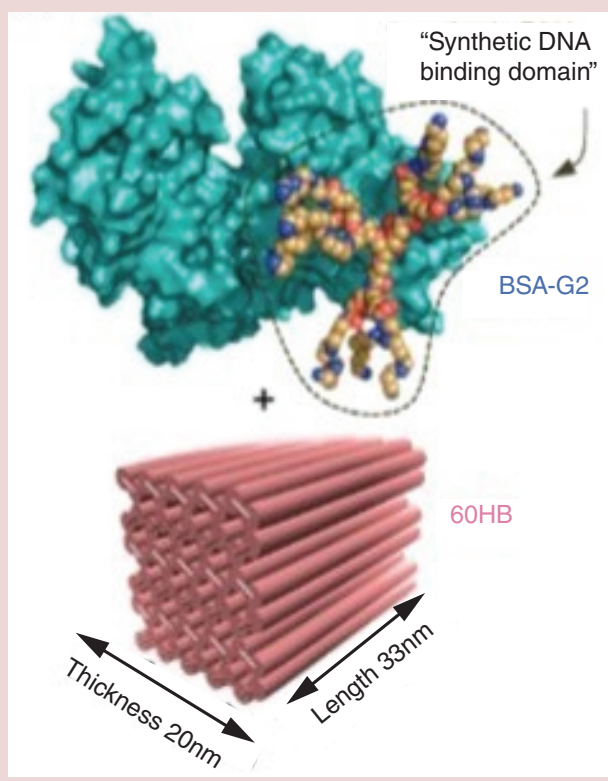

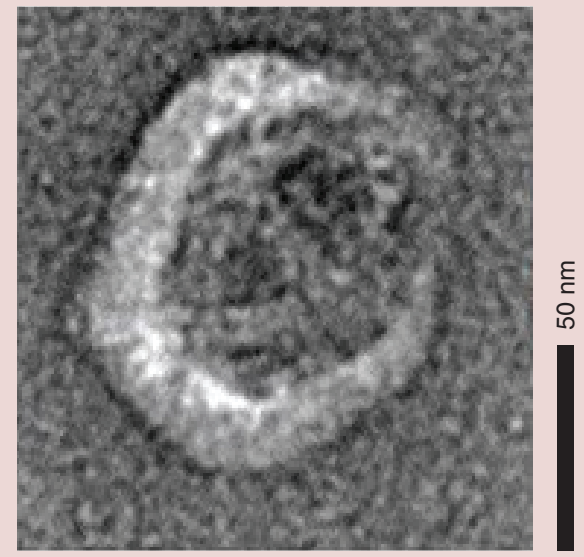
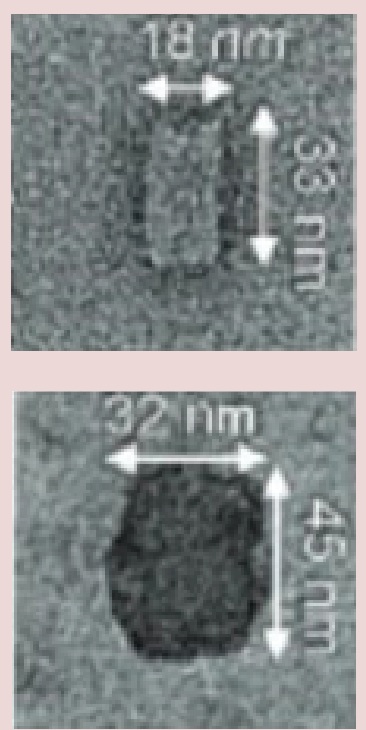

Figure 3. Increasing stability and immuno-compatibility en route to cells. (A) DNA origamis can be passivated and stabilized in in vivo conditions by encapsulating them into a lipid membrane which has been shown to dramatically increase the circulation time in blood. (B) Similarly, coating with immune inert proteins (BSA) has been achieved using protein-dendron conjugates with a synthetic DNA binding domain, which enhanced the stability and transfection rate and reduced the immunostimulation as assessed by IL-6 production. For both cases in the figure, schematics are shown on the left while TEM images of experimentally produced particles are shown on the right.

BSA: Bovine serum albumin.

(A) Reproduced with permission from [61] (c) American Chemical Society (2014),

https://pubs.acs.org/doi/abs/10.1021/nn5011914 (further permissions related to the material excerpted should be directed to the ACS).

(B) Reproduced with permission from [62] ๔ WILEY-VCH Verlag GmbH \& Co. KGaA, Weinheim (2017).

distributed throughout the body rather than concentrated in the urine, and remaining for a prolonged period in the blood.

Coating DNA origami structures with immune-inert proteins is another method for enhancing both decreased immune responses and transfection. This has been shown in work where a DNA origami was coated with BSA conjugated to synthetically produced, modified spermidine dendrons that contained protonatable amines [62]. Mixing the DNA origami with the dendron resulted in coating of the origami, which was confirmed with EMSA 
and TEM. BSA coated DNA origami was transfected in HEK293 cells and it was observed that the coating increased the transfection rate and may lead to escape of the origami from the endosome (in some cases no colocalization of origami and endosomal dye was observed). It was shown that the BSA coating dramatically diminished the immune response of mouse primary splenocytes and enhanced resistance to DNase I treatment.

\section{Endosomal avoidance/escape}

Typically, delivered nanoparticles are taken up by cells into the endosome and the challenge arises of how they and the therapeutic cargo can escape, reaching the final target. To answer this question, the ability to observe the location of DNA nanostructures in cells is necessary and has been demonstrated using tube-like DNA tile structures attached with fluorescent dye, folate modifications and siRNA for green fluorescent protein (GFP) knockdown [63]. Folate-modified as well as unmodified nanotubes were taken up by HeLa cells without notable difference and localized in the endosome (shown by colocalization of fluorescence labeled (Atto488) nanotubes and dextran staining). In agreement with this observation, addition of siRNA molecules to the tube did not lead to suppression of GFP production in GFP expressing HeLa cells, whereas lipofection with siRNA silenced the GFP signal.

Strategies to increase stability in cell culture and avoid the endosome have been specifically addressed for drugloaded origami structures. In one example [64], a rod-like DNA origami structure was designed and loaded with the anthracycline drug daunorubicin, widely used in treating myeloid leukemia. This strategy resulted in bypassing MDR-1 resistance. The produced design appeared to overcome the breakdown in serum (presumably due to nucleases) previously reported [39]. Testing of this DNA origami structure (named 'Horse') to deliver daunorubicin to HL-60 and HL-60/ADR cells (overexpressing MDR-1, making them resistant to daunorubicin) showed a sustained $40 \%$ increase in intracellular fluorescence due to the drug. Fluorescence microscopy of HL-60/ADR cells also labeled with Lysotracker Green confirmed time dependent uptake and localization to the lysosomal compartment and the signal increased for $5 \mathrm{~h}$ when steady state was reached. Unloaded Horse was also internalized but did not kill cells while loaded Horse did result in cell death. It was hypothesized that endosomal uptake bypassed efflux pumps and that the low $\mathrm{pH}$ in the endosome leads to release of the drug. Loaded Horse appeared not to increase the cytotoxicity of the drug but rather caused increased inhibition of cell growth. This is consistent with the mechanism of action of daunorubicin which is cell growth inhibition. Overall, the results support a model whereby loaded origami causes improved retention.

Direct delivery to the cytosol is another approach to avoid the endosome. This has been achieved recently using a 'cell squeezing' technique (Figure 4) [65] applicable in vitro. Here, a standard DNA rectangle with attached proteins was successfully delivered.

In the squeezing technique, cells are passed through a device wherein they undergo constriction, which results in the temporary development of small holes in the membrane. Circulating particles such as protein/DNA or RNA are able to pass through these holes and enter the cell [66]. The advantage of cell squeezing is that it appears to be less disruptive to cells than other permeabilization techniques while the advantage of delivering proteins attached to DNA origami rather than naked proteins is that this method appears to be more efficient than using proteins alone. In addition, attaching proteins to DNA origami allows precise control over the relative stoichiometry of delivered proteins. The work showed that functional proteins attached to DNA origami could be delivered to the cell and also that addition of a nuclear localization sequence to the DNA origami resulted in it being delivered to the nucleus, something only seen in cell squeezing, not in endocytosis.

\section{Subcellular localization}

DNA origami structures delivered successfully to cells subsequently have to proceed to the correct subcellular location (Figure 5). Techniques for visualizing subcellular localization have been developed. In one example [67], a carbazole-based biscyanine was used to label formed DNA origami structures - avoiding the need to purchase fluorescently labeled DNA. The molecule is not significantly fluorescent until it is bound to DNA meaning that breakdown of DNA (loss of fluorescence) can be monitored. Delivery of such labeled DNA origami to cells was carried out and the resulting signal showed them to be localized in/around the lysosome. Stability could also be assessed, with a significant decrease in signal seen at $24-48 \mathrm{~h}$ and loss of signal by $60 \mathrm{~h}$.

One intriguing possibility to overcome problems of reaching and entering cells is direct production of DNA nanostructures in the cytoplasm. Indeed, the enzymatic production of ssDNA and its assembly into a simple DNA nanostructure has been demonstrated in Escherichia coli [68]. Moreover, a synthetic scaffold for DNA origami has been produced in this way [69]. The in silico design was achieved by means of a custom-made CaDNAno plugin. One 


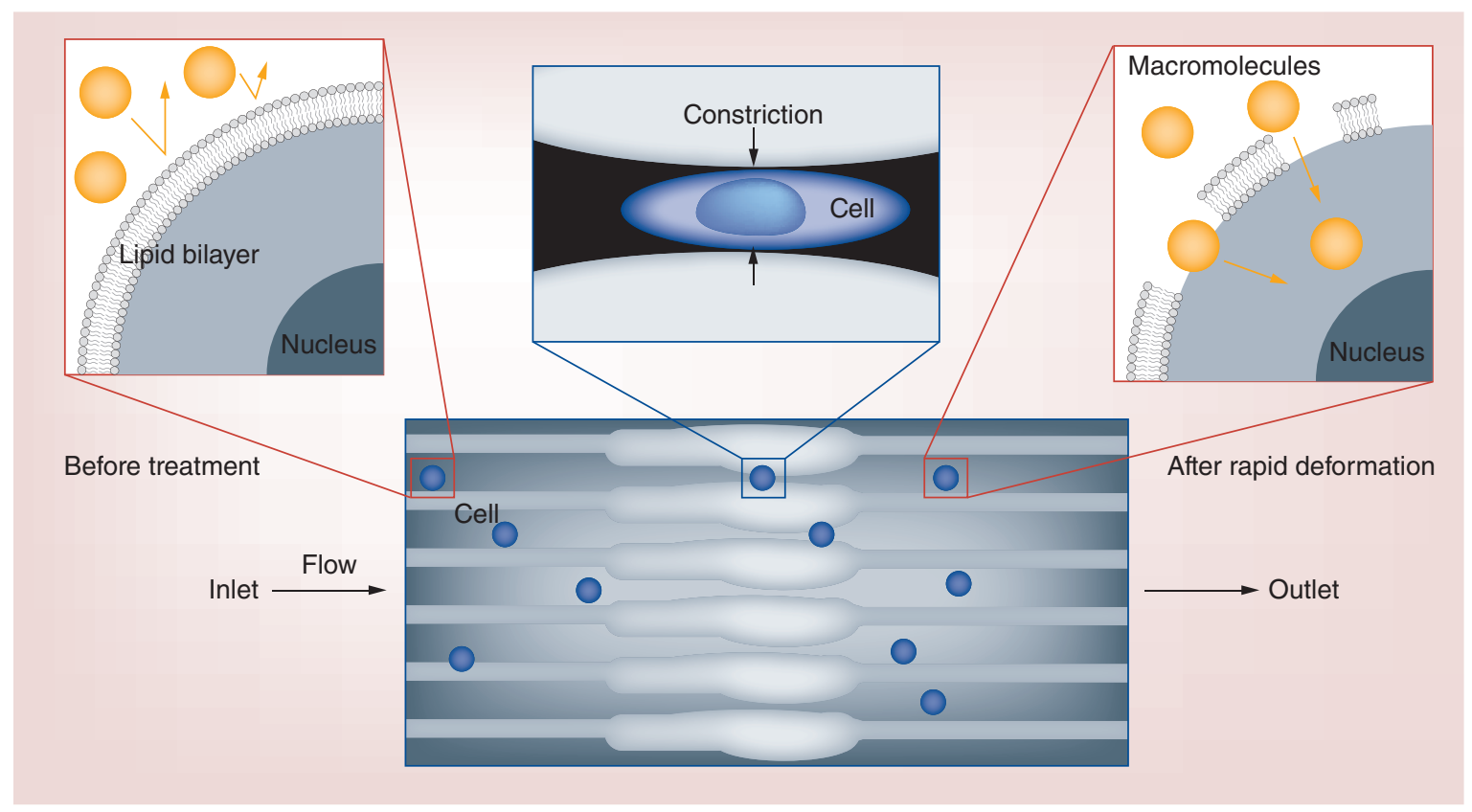

Figure 4. Schematic of the cell squeezing technique. While cells undergo constriction, transient holes develop that allow particles to enter the cell.

Reproduced with permission from [66] (c) National Academy of Sciences (2013).

advantage of synthetic scaffolds is removal of sub-sequence repetitions, which should reduce misfolding, as kinetic traps are avoided. In addition, the synthetic scaffold was constructed to be 'bio-orthogonal', as no known genetic sequences were found in the scaffold using NCBI BLAST. For validation, the synthetic scaffold was produced by amplification and folded into a square shaped DNA origami. One of the synthetic scaffolds produced in the study was successfully folded in an RNA-DNA hybrid origami. Following previous work [70], the $1000 \mathrm{nt}$ long RNA scaffold was produced by in vitro transcription and folded into a triangular structure by the help of DNA-staple strands [69].

\section{In-cell functionality}

The power of DNA origami lies in its ability to embody a range of functionality from straightforward drug loading to targeting and programmable robotics. Demonstration of functionality within cells, particularly those requiring complex interactions and/or conformational changes is of great interest and several examples have demonstrated intracellular DNA origami robotic activity (Figure 6). One work [71] describes a 3D DNA nanorobot, which is capable of opening and closing a flap in the outer wall of a hollow capsular structure in response to a signal from outside, whereby base pairing to an added DNA strand acts as a nano-actuator, 'pulling' the flap open. When a small amount of target DNA is present, the flap is opened exposing a hemin binding nucleotide sequence to the external solution. This leads to hemin (present in the external solution) being bound by the sequence, resulting in the formation of a horseradish peroxidase mimicking DNAzyme, which could be detected by a colorimetric oxidation assay.

To date, a major focus of intracellular functionality for DNA origami has been using it to affect immunomodulation: for example, a DNA origami tube was constructed and decorated with multiple external, unmethylated CpG sequences that are typical for microbial DNA and known to be immunostimulatory [72]. Cellular uptake tests showed that the DNA origami structures were taken up by splenic macrophages and were localized to the endosome. In general, large, dense structures were taken up better than smaller, less dense structures. The structures were indeed found to be immunostimulatory compared with controls such as unmodified tubes or short CpG containing sequences alone. Immune stimulation was assessed by measuring IL-6 secretion and expression of CD69. Origami tubes lacking CpG on the surface showed only a mild production of IL-6. However, the undecorated DNA origami tubes showed intermediate stimulation of CD69 expression on dendritic cells and B-lymphocytes. This ability of unmodified DNA origami structures to moderately activate innate immunity is important as it may 
Figure 5. Subcellular localization and in vivo imaging of DNA origami structures. (A) Double-stranded DNA origami can bind to a biscyanine label and form an origami-label complex with strong emission. (B) After forming the complex, DNA origami can be administered to cells and its intracellular location and degradation can be detected via fluorescence microscopy. (C) Exemplary confocal images of MCF-7 cells, showing the localization of DNA origami-label complex in lysosomes (blue: nucleus, red: lysosome, green: DNA origami-probe complex).

Reproduced with permission from [67] (c) Royal Society of Chemistry (2012).

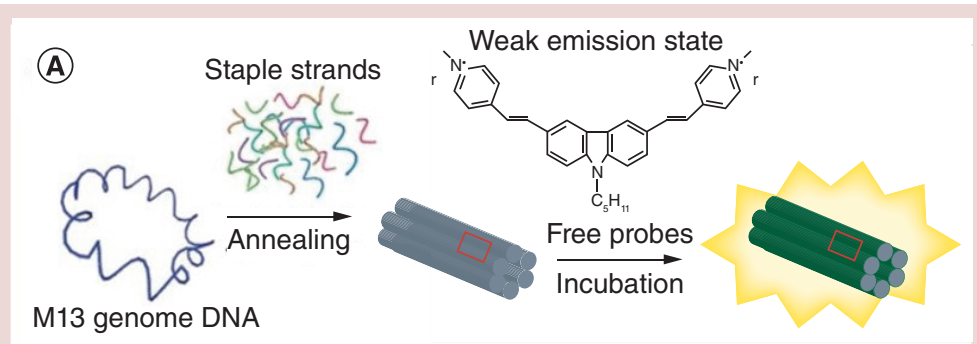

(B)

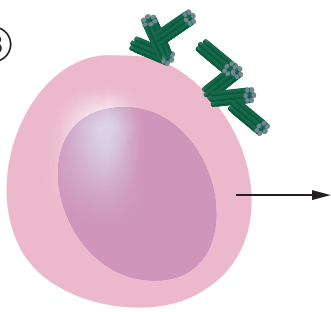

Cell uptake of origami-probe complex

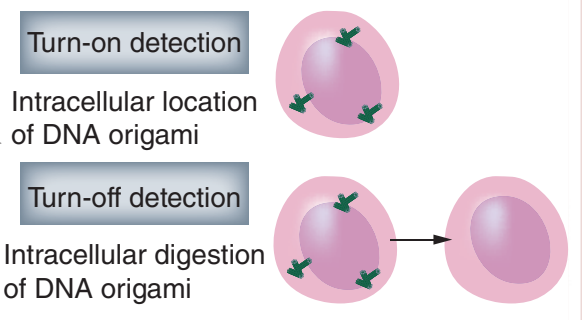

(C) Bright field

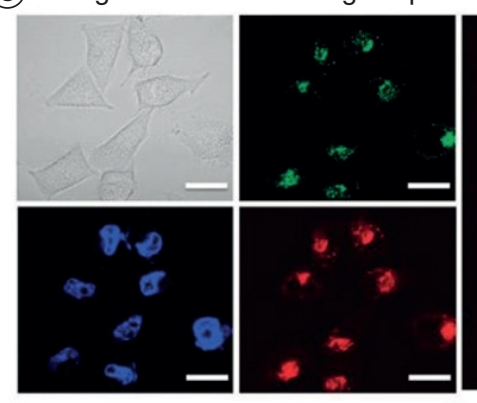

Hoechst 33342

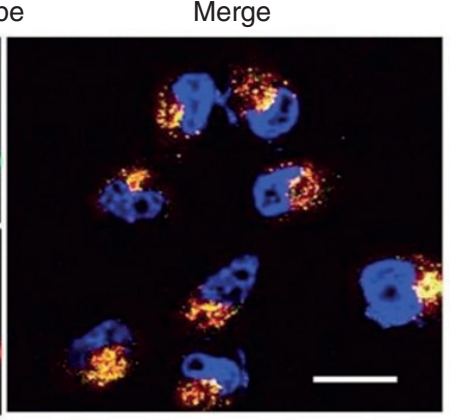

LysoTracker red

be a barrier to the use of DNA origami in vivo. A model of origami update into endosome followed by disassembly and stimulation by the CpG of the TLR9 receptor was suggested.

Another area of in-cell DNA origami functionality is the delivery of small molecule drugs, with DNA intercalators being particularly suitable. A DNA origami triangle intercalated with dox and a DNA origami tube and triangle also loaded with dox have been tested for internalization and killing in MCF-7 cells [44]. The loaded DNA origami structures killed regular MCF-7 cells with similar efficacy as dox alone but showed significantly increased toxicity against dox resistant MCF-7, while DNA origami without drug showed no cytotoxicity. Confocal microscopy studies also seemed to suggest DNA origami is able to increase intracellular accumulation of dox in resistant cells with DNA origami structures shown to be localized in the lysosomes (at $6 \mathrm{~h}$ ) and dox loaded DNA origami in resistant cells led to an inhibition of lysosomal acidification hypothesized to allow the drug to distribute out of the lysosome. Time-lapse live cell imaging of different dox-loaded DNA origami shapes (2D crosses, rectangles and $3 \mathrm{D}$ triangles) enabled a comparison of the effect of shape on cellular uptake, with the 3D triangle apparently being most effective [74]. Dox delivery via DNA origami was also demonstrated in another report where two DNA origamis having different degrees of twist (straight nanotube and twisted nanotube) were designed and tested with regard to dox intercalation and cytotoxicity toward different breast cancer cell lines (MDA-MB-231, MDA-MB468 and MCF-7) [22]. The twisted nanotube was found to be more compact in the presence of dox and to fold with higher yield, whereas the straight tube showed poorer folding with increasing dox concentrations. Moreover, the twisted nanotube structure had a higher loading capacity and showed slower release compared with straight nanotubes. Cytotoxicity was examined using the sulforhodamine B calorimetric assay. After $48 \mathrm{~h}$ of incubation, the half-maximal inhibitory concentration for dox was significantly lower when delivered intercalated in twisted nanotube structures (dox/T-Nano). Confocal microscopy of labeled dox/T-Nano showed efficient delivery of dox 
(A)

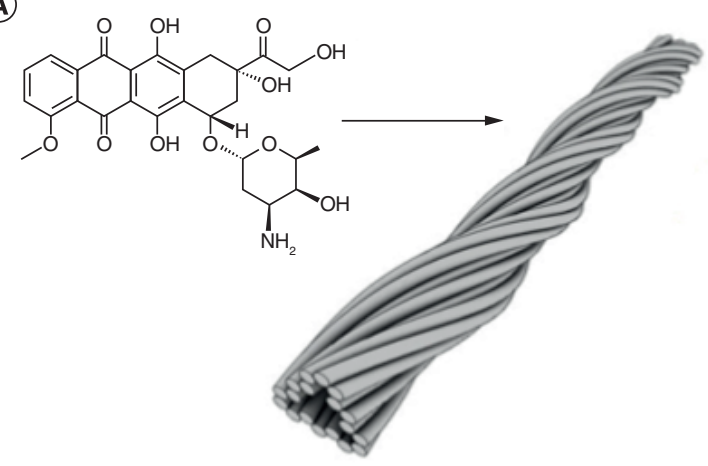

(B)

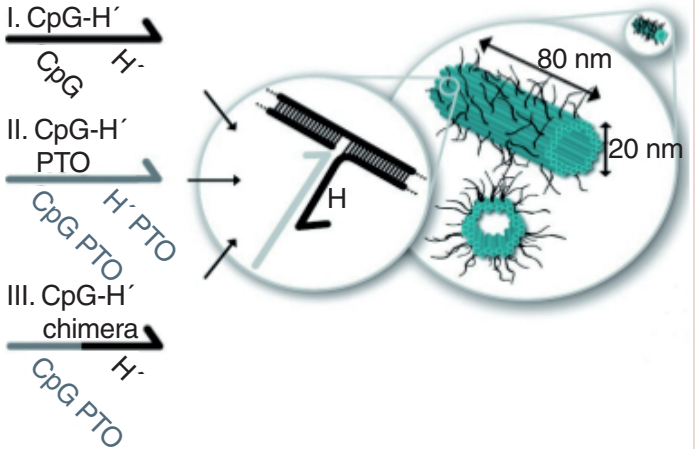

(C)

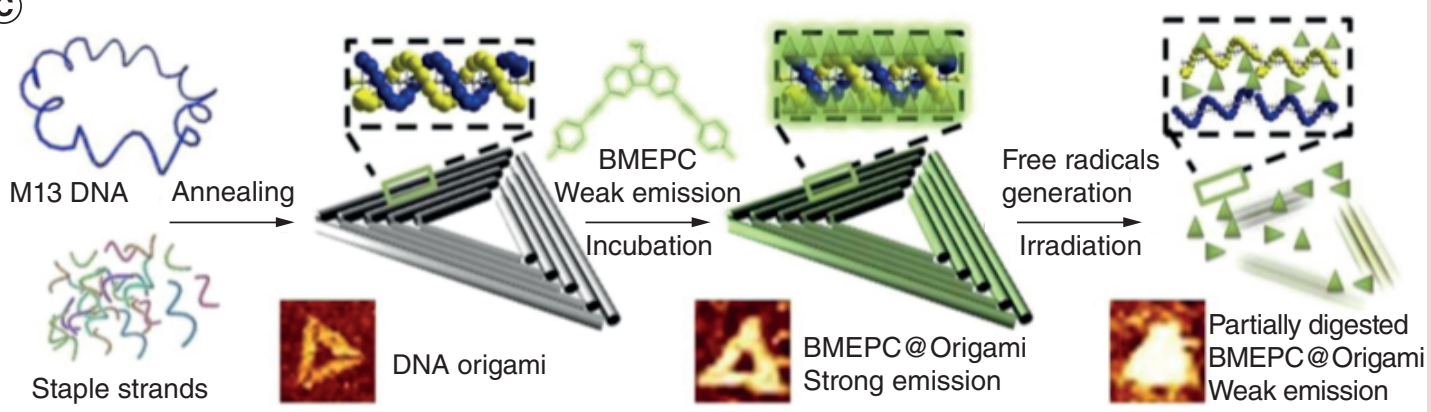

Figure 6. DNA origami structures and their applications inside the cell. (A) DNA origami tube structure loaded with dox. (B) DNA origami tube bearing CpG handle extensions with and without phosphorothioate. (C) Preparation of triangular shaped DNA origami loaded with BMEPC. All structures are internalized by endocytic pathways. dox: Doxorubicin.

(A) Reproduced with permission from [22] (c) American Chemical Society (2012),

https://pubs.acs.org/doi/abs/10.1021/nn3022662 (further permissions related to the material excerpted should be directed to the ACS).

(B) Reproduced with permission from [72] ๔ American Chemical Society (2011).

(C) Reproduced with permission from [73] ® American Chemical Society (2016).

to the cell nuclei via degradation of the DNA origami structure in the endosomes. FACS analysis showed that the dox/T-Nano structures cause a considerable increase in the apoptosis of cancer cells.

DNA origami has even been used as a protective cage for delivery of a functional enzyme: luciferase has been attached to the inside of an open-ended DNA origami barrel which was delivered to HEK293 cells [75] with cells exposed to enzyme loaded into origami showing significantly higher luminescence than those exposed to enzyme alone as assessed by confocal microscopy.

Photothermal therapy using gold nanoparticles mediated by DNA origami structures has been demonstrated: it is known that gold nanoparticles can absorb laser light leading to rapid heating resulting in a decrease of the viability of surrounding cells. This has been exploited for thermal therapy in cancer [76]. In one work, a DNA origami tube and a triangle were attached to gold nanorods approximately $40 \mathrm{~nm}$ in length with the aim of using them for both diagnostics and photothermal therapy of tumors [77]. To observe in vitro internalization, goldorigami structures or gold nanoparticles alone were incubated for $24 \mathrm{~h}$ with MCF-7 cells and visualized by using two-photon luminescence imaging. Cellular entry of gold particles attached to origami was significantly enhanced compared with gold alone. Between origami structures, the triangle showed better internalizing properties and was subsequently used for in vitro and in vivo phototherapy experiments. In in vitro experiments using MCF-7 cells as well as in vivo experiments using an MCF-7 xenograft tumor model, triangle gold complexes showed enhanced efficiency for photothermal therapy compared with free gold nanoparticles alone (better killing of MCF-7 cells, higher tumor temperature after photothermal therapy and higher survival rates in the xenograft model). 
In another report, light irradiation to produce free radicals was demonstrated using an intercalator [73]: here, the photosensitive agent BMEPC was loaded by intercalating into the DNA origami structure. Incubation of the resulting DNA origami-BMEPC complex with MCF-7 cells led to internalization. Subsequent light irradiation led to production of free radicals and singlet oxygen by BMEPC, which killed the breast cancer cells and simultaneously destroyed the DNA origami structure through photocleavage.

Origami-mediated control of transcription has also been shown [78], in work where a DNA binding domain and a transcription activator domain were placed on opposite sides of a DNA origami rod. After validating the structure and its binding to both proteins, it was microinjected into the yolks of single-celled zebrafish embryos. Six hours after injection, the embryos were analyzed by means of microscopy. No toxic effect of the origami injection could be observed. Cy5 labeled DNA origami was found to accumulate mainly in the cytosol of many of the developing cells, suggesting good internalization of the structure from the yolk. The aim was to control gene activation using the DNA origami conjugated to the DNA binding and transcriptional activator domain. However, as the origami was not able to reach the nucleus, selective gene activation in vivo could not be readily achieved. In the future stronger nuclear localization signals could be used to achieve this goal.

\section{Conclusion \& future perspective}

DNA origami offers a new system of delivering nanoparticles to cells. While there are other more mature technologies already available to do this (such as liposomes of which many are approved for clinical use [79]) origami has the potential to offer number of advantages namely: i) monodipsersity - a given, successfully designed DNA origami should in principle always fold to a precise structure though of course some variations can arise; ii) small size - once folded, DNA origami structures typically have diameters in the tens of nanometers size range, smaller than many other delivery systems including most liposomes and are at a similar size regime as small virus nanoparticles; iii) addressability and designability leading to programmable functionality - DNA origami includes a large number of staple strands each of which can be individually addressed, allowing the possibility of multiple further molecules (such as proteins or other molecules with therapeutic potential) being attached at chosen locations. This is a useful feature found in few if any other systems. Using attached molecules to control conformational changes in DNA origami structures further lends itself to construction of programmable systems for example, where combinations of binding events trigger changes such as exposure/release of cargoes. Such programmable 'robotics' is another feature where DNA origami has great potential compared with other systems.

A major set of challenges remain apart from ensuring the therapeutic functionality of DNA origamis themselves, namely to find a way that such nanoparticles can survive in the body and reach target cells intact in a manner which is efficient, biocompatible, non-toxic and nonimmunogenic, and offers advantages over existing systems. Challenges include i) immunogenicity - it is still unclear if naked DNA origami structures are sufficiently immune silent to be usable and some form of immune response without passivation can be expected [80]; ii) Stability in the body as outlined above, progress has been made in producing DNA origamis whose stability is more compatible with conditions found in the body/cells but more rigorous in vivo demonstrations of particular systems will be necessary to prove their usefulness; iii) widening of cargo-carrying capability - to date, most therapeutic molecules shown to be able to be carried and released by DNA origamis have been small DNA interchelators. Further demonstrations of successful capture, delivery and release of more diverse therapeutics is desirable; iv) expense - typically, DNA origami structures contain around 200 staple strands of a few tens of bases in length. These are produced synthetically and while DNA synthesis costs are decreasing, it is still the case that producing DNA origami is expensive, especially so if large amounts necessary for industrial/clinical use are required. Some progress has recently been made in this area where biotechnological methods for DNA origami staple strand production have been demonstrated [81]. These challenges are significant and DNA origami is still at the earliest phase of development in terms of in vivo functionality. The work summarized here represents the current state of development and hints at future areas where progress is needed if a DNA origami-based therapeutic is to be realized. If these challenges can be met then there is a realistic prospect that DNA origami structures may be usable as highly specific drug delivery systems and even beyond, as 'machines' that direct cellular processes along desired routes.

Financial \& competing interests disclosure

The authors are funded by the Team programme of the Foundation for Polish Science co-financed by the European Union under the European Regional Development Fund (TEAM/2016-3/23). The authors have no other relevant affiliations or financial involvement 
Executive summary

DNA origami has potential as a smart therapeutic

- DNA origami allows a long strand of DNA to be folded into complex shapes by numerous smaller staple strands.

- Produced DNA shapes can be programmed and can carry therapeutic cargos, that is, can act as 'nanorobots'.

- Specific delivery to and functionality in cells is still a challenge.

Methods are being developed to target DNA origami to cells \& stimulate uptake

- Ligands decorating DNA origami to target specific cell types have been developed and include folate for targeting the folate receptor which is overexpressed on many cancer cells.

- DNA aptamer 'locks' and 'keys' can be employed to control opening/closing of DNA origami containers only in the presence of desired ligands for example, specific cell-surface receptors such as MUC1.

- Decoration of DNA origami with cell-penetrating molecules may allow entry into cells even in the absence of the ability to bind a specific receptor, for example, cell-penetrating peptides.

- Direct delivery to the cytoplasm can be achieved via electrotransfection.

- Methods have been developed to allow DNA origami to retain integrity in the lower $\mathrm{Mg}^{2+}$ conditions found physiologically.

- Coating of DNA origami with immune-silent materials may help to reduce undesirable immune-stimulation.

Delivered DNA origami must avoid/escape the endosome

- Delivered nanoparticles are commonly taken up into endosomes.

- Small molecules carried by DNA origami can escape from the endosome.

- Endosomal uptake can be bypassed if a cell-squeezing technique for delivery to the cytosol is used.

DNA origami subcellular localization is challenging to achieve \& observe

- Fluorescent labeling such as using carbazole-based biscyanine can be used.

- Intracellular production and folding of DNA nanostructures is a possible way to overcome many of the obstacles to reach the cell interior but is difficult to achieve.

A range of in-cell functionalities is possible for DNA origami

- A hollow DNA nanorobot capsule has been demonstrated, having a flap in the capsule wall that can be programmably opened, leading to hemin binding and a colorimetric signal.

- Decoration with CPG motifs leads to increased immunostimulation.

- Small drug molecules can be delivered to cells and DNA-chelating molecules such as doxorubicin are particularly suitable because of their inherent ability to bind double-stranded DNA-based structures.

- Attaching gold nanoparticles to DNA origami structures allows cell-killing via photothermal therapy after cell uptake.

- Photosensitive intercalating agents such as BMEPC can also be loaded on DNA origami structures and irradiated after cell-uptake leading to free-radical production and cell-death.

Conclusion \& future perspective

- The high designability and programmability of DNA origami gives it the potential to be used as a sophisticated robotic therapeutic.

- But to achieve this, questions including in vivo stability, targetability and immune-modulation need to be addressed.

- If the questions can be satisfactorily addressed, it may be possible to use DNA origamis as sophisticated drug delivery systems or even to control cell-cycle processes.

with any organization or entity with a financial interest in or financial conflict with the subject matter or materials discussed in the manuscript apart from those disclosed.

No writing assistance was utilized in the production of this manuscript.

\section{Open access}

This work is licensed under the Attribution-NonCommercial-NoDerivatives 4.0 Unported License. To view a copy of this license, visit http://creativecommons.org/licenses/by-nc-nd/4.0/ 


\section{References}

Papers of special note have been highlighted as: $\bullet$ of interest; $\bullet \bullet$ of considerable interest.

1. Seeman NC. Nucleic acid junctions and lattices. J. Theor. Biol. 99(2), 237-247 (1982).

2. Kallenbach NR, Ma RI, Seeman NC. An immobile nucleic acid junction constructed from oligonucleotides. Nature 305(5937), 829-831 (1983).

3. Wang Y, Mueller JE, Kemper B, Seeman NC. Assembly and characterization of five-arm and six-arm DNA branched junctions. Biochemistry 30(23), 5667-5674 (1991).

4. Wang X, Seeman NC. Assembly and characterization of 8-arm and 12-arm DNA branched junctions. J. Am. Chem. Soc. 129(26), 8169-8176 (2007).

5. Chen J, Seeman NC. Synthesis from DNA of a molecule with the connectivity of a cube. Nature 350(6319), 631-633 (1991).

6. Zhang Y, Seeman NC. Construction of a DNA-truncated octahedron. J. Am. Chem. Soc. 116(5), 1661-1669 (1994).

7. Winfree E, Liu F, Wenzler LA, Seeman NC. Design and self-assembly of two-dimensional DNA crystals. Nature 394(6693), 539-544 (1998).

8. Goodman RP, Berry RM, Turberfield AJ. The single-step synthesis of a DNA tetrahedron. Chem. Commun. 0(12), 1372-1373 (2004).

9. Goodman RP, Schaap IA, Tardin CF et al. Rapid chiral assembly of rigid DNA building blocks for molecular nanofabrication. Science 310(5754), 1661-1665 (2005).

10. Kim KR, Kim DR, Lee T et al. Drug delivery by a self-assembled DNA tetrahedron for overcoming drug resistance in breast cancer cells. Chem. Commun. 49(20), 2010-2012 (2013).

11. Lee H, Lytton-Jean AK, Chen Y et al. Molecularly self-assembled nucleic acid nanoparticles for targeted in vivo siRNA delivery. Nat. Nanotechnol. 7(6), 389-393 (2012).

12. Liang L, Li J, Li Q et al. Single-particle tracking and modulation of cell entry pathways of a tetrahedral DNA nanostructure in live cells. Angew. Chem. Int. Ed. Engl. 53(30), 7745-7750 (2014).

13. Liu X, Xu Y, Yu T et al. A DNA nanostructure platform for directed assembly of synthetic vaccines. Nano Lett. 12(8), 4254-4259 (2012).

14. Rothemund PW. Folding DNA to create nanoscale shapes and patterns. Nature 440(7082), 297-302 (2006).

-. Introduces the DNA origami method for the creation of nanoscale objects made of DNA.

15. Wang P, Meyer TA, Pan V, Dutta PK, Ke Y. The beauty and utility of DNA origami. Chem 2(3), 359-382 (2017).

16. Castro CE, Kilchherr F, Kim DN et al. A primer to scaffolded DNA origami. Nat. Methods 8(3), 221-229 (2011).

17. Hong F, Zhang F, Liu Y, Yan H. DNA origami: scaffolds for creating higher order structures. Chem. Rev. 117(20), 12584-12640 (2017).

18. Shih WM, Lin C. Knitting complex weaves with DNA origami. Curr. Opin. Struct. Biol. 20(3), 276-282 (2010).

19. Douglas SM, Marblestone AH, Teerapittayanon S, Vazquez A, Church GM, Shih WM. Rapid prototyping of 3D DNA-origami shapes with caDNAno. Nucleic Acids Res. 37(15), 5001-5006 (2009).

-. Introduces software for the design of complex DNA origami structures.

20. Wagenbauer KF, Engelhardt FAS, Stahl E et al. How we make DNA origami. Chembiochem 18(19), 1873-1885 (2017).

21. Zhang Q, Jiang Q, Li N et al. DNA origami as an in vivo drug delivery vehicle for cancer therapy. ACS Nano 8(7), 6633-6643 (2014).

22. Zhao YX, Shaw A, Zeng X, Benson E, Nyström AM, Högberg B. DNA origami delivery system for cancer therapy with tunable release properties. ACS Nano 6(10), 8684-8691 (2012).

- Demonstrates the use of DNA origami for delivering drugs to cancer cells.

23. Meyer R, Giselbrecht S, Rapp BE, Hirtz M, Niemeyer CM. Advances in DNA-directed immobilization. Curr. Opin. Chem. Biol. 18, $8-15$ (2014).

24. Angelin A, Weigel S, Garrecht R et al. Multiscale origami structures as interface for cells. Angew. Chem. Int. Ed. Engl. 54(52), 15813-15817 (2015)

25. Linko V, Ora A, Kostiainen MA. DNA nanostructures as smart drug-delivery vehicles and molecular devices. Trends Biotechnol. 33(10), 586-594 (2015).

26. Vinther M, Kjems J. Interfacing DNA nanodevices with biology: challenges, solutions and perspectives. New J. Phys. 18(8), 1-9 (2016).

27. Udomprasert A, Kangsamaksin T. DNA origami applications in cancer therapy. Cancer Sci. 108(8), 1535-1543 (2017).

28. Smith D, Schüller V, Engst C, Rädler J, Liedl T. Nucleic acid nanostructures for biomedical applications. Nanomedicine 8(1), 105-121 (2013).

29. Okholm AH, Kjems J. DNA nanovehicles and the biological barriers. Adv. Drug Deliv. Rev. 106, 183-191 (2016).

30. Weissleder R, Nahrendorf M, Pittet MJ. Imaging macrophages with nanoparticles. Nat. Mater. 13(2), 125-38 (2014).

31. Rejman J, Oberle V, Zuhorn IS, Hoekstra D. Size-dependent internalization of particles via the pathways of clathrin- and caveolae-mediated endocytosis. Biochem. J. 377(1), 159-169 (2004). 
32. Bianco A, Kostarelos K, Prato M. Applications of carbon nanotubes in drug delivery. Curr. Opin. Chem. Biol. 9(6), 674-679 (2005).

33. Godonoga M, Lin TY, Oshima A et al. A DNA aptamer recognising a malaria protein biomarker can function as part of a DNA origami assembly. Sci. Rep. 6, 21266 (2016).

34. Amir Y, Ben-Ishay E, Levner D, Ittah S, Abu-Horowitz A, Bachelet I. Universal computing by DNA origami robots in a living animal. Nat. Nanotechnol. 9(5), 353-357 (2014).

35. Mei Q, Wei X, Su F et al. Stability of DNA origami nanoarrays in cell lysate. Nano Lett. 11(4), 1477-1482 (2011).

36. Neumann E, Schaefer-Ridder M, Wang Y, Hofschneider PH. Gene transfer into mouse lyoma cells by electroporation in high electric fields. EMBO J. 1(7), 841-845 (1982).

37. Heller LC, Heller R. In vivo electroporation for gene therapy. Hum. Gene Ther. 17(9), 890-897 (2006).

38. Kielar C, Xin Y, Shen B et al. On the Stability of DNA origami nanostructures in low-magnesium buffers. Angew. Chem. Int. Ed. 57(30), 9470-9474 (2018).

39. Hahn J, Wickham SFJ, Shih WM, Perrault SD. Addressing the Instability of DNA nanostructures in tissue culture. ACS Nano 8(9), 8765-8775 (2014).

40. Chopra A, Krishnan S, Simmel FC. Electrotransfection of polyamine folded DNA origami structures. Nano Lett. 16(10), 6683-6690 (2016).

41. Benson E, Mohammed A, Gardell J et al. DNA rendering of polyhedral meshes at the nanoscale. Nature 523(7561), 441-444 (2015).

42. Zhu G, Zheng J, Song E et al. Self-assembled, aptamer-tethered DNA nanotrains for targeted transport of molecular drugs in cancer theranostics. Proc. Natl Acad. Sci. USA 110(20), 7998-8003 (2013).

43. Qu Y, Yang J, Zhan P et al. Self-assembled DNA dendrimer nanoparticle for efficient delivery of immunostimulatory CpG motifs. ACS Appl. Mater. Interfaces 9(24), 20324-20329 (2017).

44. Jiang Q, Song C, Nangreave J et al. DNA origami as a carrier for circumvention of drug resistance. J. Am. Chem. Soc. 134(32), 13396-13403 (2012).

- Demonstrates the use of DNA origami for drug delivery to cancer cells.

45. Bastings MMC, Anastassacos FM, Ponnuswamy N et al. Modulation of cellular uptake of DNA origami through control over mass and shape. Nano Lett. 18(6), 3557-3564 (2018).

46. Ellington AD, Szostak JW. In vitro selection of RNA molecules that bind specific ligands. Nature 346(6287), 818-822 (1990).

47. Tuerk C, Gold L. Systematic evolution of ligands by exponential enrichment: RNA ligands to bacteriophage T4 DNA polymerase. Science 249(4968), 505-510 (1990).

48. Bock LC, Griffin LC, Latham JA, Vermaas EH, Toole JJ. Selection of single-stranded DNA molecules that bind and inhibit human thrombin. Nature 355(6360), 564-566 (1992).

49. Cheung YW, Kwok J, Law AW, Watt RM, Kotaka M, Tanner JA. Structural basis for discriminatory recognition of Plasmodium lactate dehydrogenase by a DNA aptamer. Proc. Natl Acad. Sci. USA 110(40), 15967-15972 (2013).

50. Tang MSL, Shiu SC, Godonoga M et al. An aptamer-enabled DNA nanobox for protein sensing. Nanomedicine 14(4), 1161-1168 (2018).

51. Rinker S, Ke Y, Liu Y, Chhabra R, Yan H. Self-assembled DNA nanostructures for distance-dependent multivalent ligand-protein binding. Nat. Nanotechnol. 3(7), 418-422 (2008).

52. Sakai Y, Islam MS, Adamiak M, Shiu SCC, Tanner JA, Heddle JG. DNA aptamers for the functionalisation of DNA origami nanostructures. Genes 9(12), 571 (2018)

53. Shiu SCC, Kinghorn AB, Sakai Y, Cheung YW, Heddle JG, Tanner JA. The three s's for aptamer-mediated control of DNAnanostructure dynamics: shape, self-complementarity, and spatial flexibility. Chembiochem 19(18), 1900-1906 (2018).

54. Chaithongyot S, Chomanee N, Charngkaew K, Udomprasert A, Kangsamaksin T. Aptamer-functionalized DNA nanosphere as a stimuli-responsive nanocarrier. Mater. Lett. 214, 72-75 (2018).

55. Shangguan D, Li Y, Tang Z et al. Aptamers evolved from live cells as effective molecular probes for cancer study. Proc. Natl Acad. Sci. USA 103(32), 11838-11843 (2006).

56. Shangguan D, Cao Z, Meng L et al. Cell-specific aptamer probes for membrane protein elucidation in cancer cells. J. Proteome Res. 7(5), 2133-2139 (2008).

57. Assaraf YG, Leamon CP, Reddy JA. The folate receptor as a rational therapeutic target for personalized cancer treatment. Drug Resist. Updat. 17(4-6), 89-95 (2014).

58. Mikkilä J, Eskelinen AP, Niemelä EH et al. Virus-encapsulated DNA origami nanostructures for cellular delivery. Nano Lett. 14(4), 2196-2200 (2014).

59. Vivès E, Brodin P, Lebleu B. A truncated HIV-1 Tat protein basic domain rapidly translocates through the plasma membrane and accumulates in the cell nucleus. J. Biol. Chem. 272(25), 16010-16017 (1997). 
60. Yan J, Hu C, Wang P et al. Growth and origami folding of DNA on nanoparticles for high-efficiency molecular transport in cellular imaging and drug delivery. Angew. Chemie Int. Ed. Engl. 54(8), 2431-2435 (2015).

61. Perrault SD, Shih WM. Virus-inspired membrane encapsulation of DNA nanostructures to achieve in vivo stability. ACS Nano 8(5), 5132-5140 (2014).

- Demonstrates a method to stabilize DNA origami structures in the body by lipid coating.

62. Auvinen $\mathrm{H}$, Zhang H, Nonappa et al. Protein coating of DNA nanostructures for enhanced stability and immunocompatibility. Adv. Healthc. Mater. 6(18), 1700692 (2017).

- Demonstrates a method to stabilize DNA origami structures in the body by protein-coating.

63. Kocabey S, Meinl H, MacPherson I et al. Cellular uptake of tile-assembled DNA nanotubes. Nanomaterials 5(1), 47-60 (2015).

64. Halley PD, Lucas CR, McWilliams EM et al. Daunorubicin-loaded DNA origami nanostructures circumvent drug-resistance mechanisms in a leukemia model. Small 12(3), 308-320 (2016).

65. Nikolov PM, Koßmann KJ, Schilling A, Angelin A, Brglez J. Cytosolic delivery of large supramolecular protein complexes arranged on DNA nanopegboards. bioRxiv 1-12 (2017).

66. Sharei A, Zoldan J, Adamo A et al. A vector-free microfluidic platform for intracellular delivery. Proc. Natl Acad. Sci. USA 110(6), 2082-2087 (2013).

67. Shen X, Jiang Q, Wang J et al. Visualization of the intracellular location and stability of DNA origami with a label-free fluorescent probe. Chem. Commun. 48(92), 11301-11303 (2012).

68. Elbaz J, Yin P, Voigt CA. Genetic encoding of DNA nanostructures and their self-assembly in living bacteria. Nat. Commun. 7, 11179 (2016).

69. Kozyra J, Ceccarelli A, Torelli E et al. Designing uniquely addressable bio-orthogonal synthetic scaffolds for DNA and RNA origami. ACS Synth. Biol. 6(7), 1140-1149 (2017).

70. Wang P, Ko SH, Tian C, Hao C, Mao C. RNA-DNA hybrid origami: folding of a long RNA single strand into complex nanostructures using short DNA helper strands. Chem. Commun. 49(48), 5462-5464 (2013).

71. Torelli E, Marini M, Palmano S et al. A DNA origami nanorobot controlled by nucleic acid hybridization. Small 10(14), 2918-2926 (2014).

72. Schüller VJ, Heidegger S, Sandholzer N et al. Cellular immunostimulation by CpG-sequence-coated DNA origami structures. ACS Nano 5(12), 9696-9702 (2011).

73. Zhuang X, Ma X, Xue X et al. A photosensitizer-loaded DNA origami nanosystem for photodynamic therapy. ACS Nano 10(3), 3486-3495 (2016).

74. Zeng Y, Liu J, Yang S, Liu W, Xu L, Wang R. Time-lapse live cell imaging to monitor doxorubicin release from DNA origami nanostructures. J. Mater. Chem. B. 6(11), 1605-1612 (2018).

75. Ora A, Järvihaavisto E, Zhang H et al. Cellular delivery of enzyme-loaded DNA origami. Chem. Commun. 52(98), 14161-14164 (2016).

76. Abadeer NS, Murphy CJ. Recent progress in cancer thermal therapy using gold nanoparticles. J. Phys. Chem. C 120(9), 4691-4716 (2016).

77. Jiang Q, Shi Y, Zhang Q et al. A self-assembled DNA origami-gold nanorod complex for cancer theranostics. Small 11(38), 5134-5141 (2015).

78. Angelin A, Kassel O, Rastegar S, Strähle U, Niemeyer CM. Protein-functionalized DNA nanostructures as tools to control transcription in Zebrafish Embryos. ChemistryOpen 6(1), 33-39 (2017).

79. Torchilin VP. Recent advances with liposomes as pharmaceutical carriers. Nat. Rev. Drug Discov. 4(2), 145-160 (2005).

80. Surana S, Shenoy AR, Krishnan Y. Designing DNA nanodevices for compatibility with the immune system of higher organisms. Nat. Nanotechnol. 10(9), 741-747 (2015).

81. Praetorius F, Kick B, Behler KL, Honemann MN, Weuster-Botz D, Dietz H. Biotechnological mass production of DNA origami. Nature 552(7683), 84-87 (2017). 
